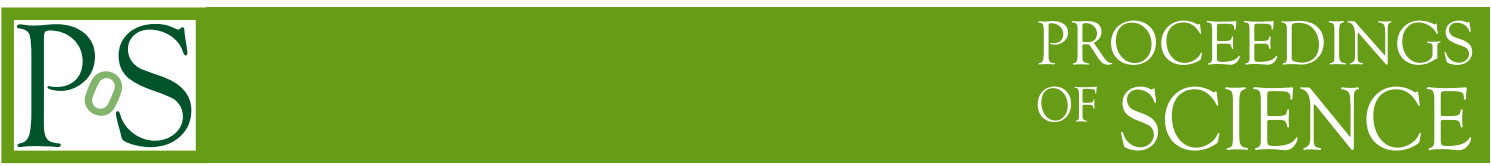

\title{
Operational experience with the ATLAS Pixel Detector at the LHC
}

\author{
Dominic HirschbühI on behalf of the ATLAS Collaboration \\ Univ. Wuppertal \\ E-mail: dominic.hirschbuehlecern.ch
}

\begin{abstract}
The Pixel Detector is the innermost detector of the ATLAS experiment at the Large Hadron Collider at CERN, providing high-resolution measurements of charged particle tracks in the high radiation environment close to the collision region. This capability is vital for the identification and measurement of proper decay times of long-lived particles such as $b$-hadrons, and thus vital for the ATLAS physics program. The detector provides hermetic coverage with three cylindrical layers and three layers of forward and backward pixel detectors. It consists of approximately 80 million pixels that are individually read out via chips bump-bonded to $1744 \mathrm{n}$-in-n silicon substrates. In this paper results from the successful operation of the Pixel Detector at the LHC will be presented, including monitoring, calibration procedures, timing optimization and detector performance.
\end{abstract}

10th International Conference on Large Scale Applications and Radiation Hardness of Semiconductor Detectors,

July 6-8, 2011

Firenze Italy 


\section{Introduction}

The ATLAS detector is built around the interaction region located at one of the interaction points of the Large Hadron Collider (LHC) at CERN and is one of two general purpose experiments designed to study physics in the new energy frontier of the LHC. At design parameters, proton-proton collisions will take place every $25 \mathrm{~ns}$ in bunches of $1.2 \cdot 10^{11}$ protons per bunch at an energy of $7 \mathrm{TeV}$ per beam for an expected instantaneous luminosity of $10^{34} \mathrm{~cm}^{-2} \mathrm{~s}^{-1}$. The physics program started in 2010 at a beam energy of $3.5 \mathrm{TeV}$ and since then the LHC is gradually increasing luminosity to meet the design parameters. The ATLAS detector must be able to cope with the high rate of particle interactions while maintaining a good energy resolution, particle identification, track pattern recognition and vertex reconstruction [1]. To accomplish this, the ATLAS tracking detector is broken up into several subsystems. The Inner Detector (ID) consists of the Pixel Detector, the SemiConductor Tracker (SCT) and the Transition Radiation Tracker (TRT) which operate in a $2 \mathrm{~T}$ solenoid magnetic field and provide tracking and particle identification. Surrounding the ID are a system of calorimeters and the outer system is a Muon Spectrometer located in a $4 \mathrm{~T}$ toroidal magnetic field.

This paper will focus on the ATLAS Pixel Detector and, in particular, the details related to the operation of the detector during the first two years of LHC running are addressed. An outline of the Pixel Detector and the readout chain will be given, followed by the operational aspects of the detector. Finally, an overview of the data taking for the 2011 run is presented including the detector status and data-taking efficiency.

\section{The ATLAS Pixel Detector}

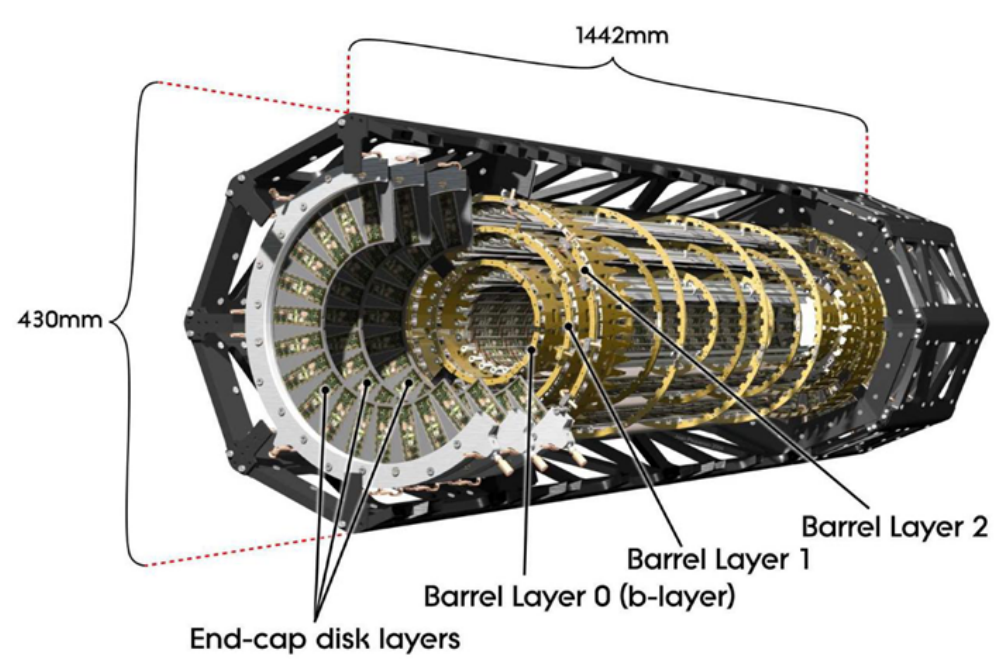

Figure 1: The ATLAS Pixel Detector. 


\subsection{Layout and structure}

The ATLAS Pixel Detector [2] is designed to provide at least three precision measurement points for tracks with pseudorapidity $|\eta|<2.5$. It is made of three concentric barrel layers and two endcaps each containing three disks, see Fig. 1. The barrel layers have radii of $50.5 \mathrm{~mm}, 88.5 \mathrm{~mm}$ and $122.5 \mathrm{~mm}$, respectively, and are $800 \mathrm{~mm}$ long. Each disk contains 48 individual modules and the disks are located at $\pm 495 \mathrm{~mm}, \pm 580 \mathrm{~mm}$ and $\pm 650 \mathrm{~mm}$ from the interaction point in the $z$ direction. The barrel layers have overlapping staves of 13 modules each which are rotated of $20^{\circ}$ to compensate for the Lorentz angle in the $2 \mathrm{~T}$ magnetic field. The disks are divided into 8 sectors of six overlapping modules each for full coverage. The 1744 modules that make up the Pixel Detector are identical, mounted on carbon fibre local supports and cover a surface area of $1.7 \mathrm{~m}^{2}$. To absorb the heat produced by the modules, an evaporative $\mathrm{C}_{3} \mathrm{~F}_{8}$ cooling incorporated into the local support structure is used. This allows an operating temperature about $-15^{\circ} \mathrm{C}$ to limit the effect of radiation damage.

\subsection{The ATLAS Pixel module}

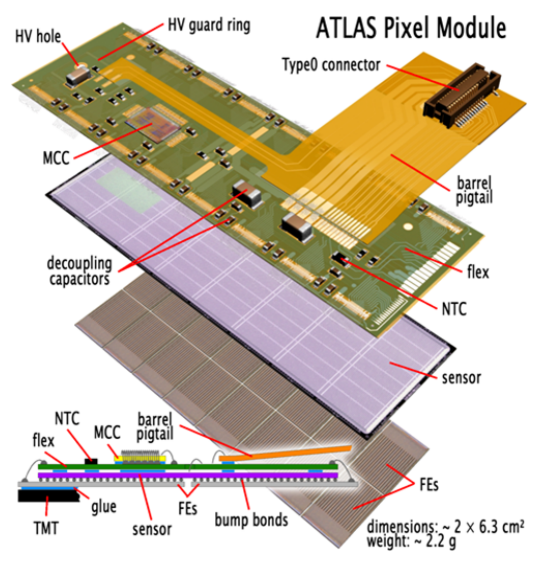

(a)

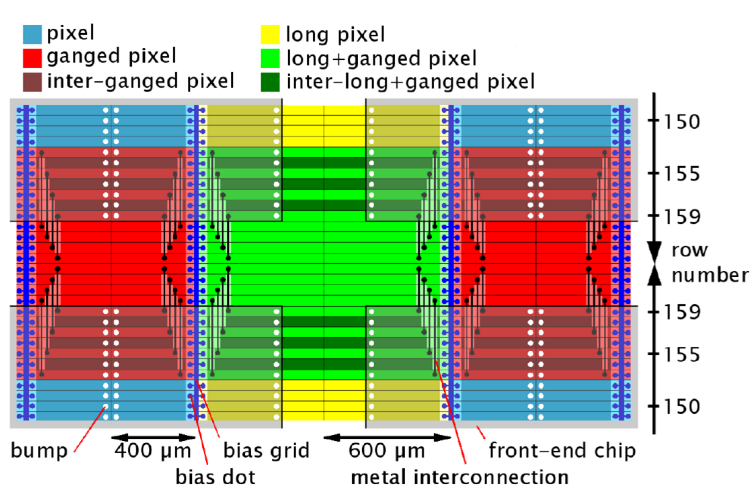

(b)

Figure 2: a) Assembly view of an ATLAS pixel module. b) End-region of a pixel sensor at the edge of four front-end chips.

The ATLAS Pixel Detector modules, see Fig. 2a, are made of a $250 \mu \mathrm{m}$ thick silicon sensor with $\mathrm{n}^{+}$pixels implanted on the $\mathrm{n}$-doped bulk with a $\mathrm{p}^{+}$backplane, 16 Front-End chips (FE-I3) and a module controller chip (MCC)[2]. The FE-I3 chips [3] were manufactured in the radiation hard $0.25 \mu \mathrm{m}$ CMOS technology and have 2880 readout channels, that match a sensor pixel size of 400 $\mu \mathrm{m} \times 50 \mu \mathrm{m}$. To enable full coverage even in the regions between front-end chips approximately $10 \%$ of the sensor pixels have a size of $600 \mu \mathrm{m} \times 50 \mu \mathrm{m}$ (long pixels). Another $2.5 \%$ of the electronics channels have two sensor pixels connected for seamless coverage in the short pixel direction (ganged pixels), see Fig. 2b. Each individual channel contains an analog and a digital circuit. The former performs pre-amplification of the collected charge, discrimination, and a measurement of the Time-over-Threshold (ToT). The latter consists of the digital readout logic to transfer hits to the peripheral circuitry of the chip, the end-of-column (EOC) logic. An 7-bit DAC for each pixel is 
used to set the discriminator threshold and counting the number of generated hits. Additionally a 5-bit DAC per FE is used to globally shift the threshold for all pixels on one FE. Zero-suppression is performed on the FE chips so that only pixels with hits are read out.

In the EOC logic hits are stored up to the programmable trigger latency and sent to the Module Control Chip (MCC) which is located on the flex board, see Fig. 2a. Together with the mere hit location and time, ToT information is read out for each hit. This is the time interval during which the preamplifier output is above the threshold, in units of the bunch crossing clock period ( $25 \mathrm{~ns}$ ). Along with providing event building and readout capabilities, the MCC routes clock, configuration data, commands and triggers to each FE. Each module has 47232 pixels for a total of $\approx 80$ million channels in the ATLAS Pixel Detector.

\subsection{Readout}

Readout Communication to the Pixel Detector is provided by an optical link which connects the off-detector Read-Out Drivers (RODs) to the modules over a distance of $80 \mathrm{~m}$. Each module has one downlink (TTC) which provides clock, trigger signals, detector calibration data and commands transmitted at $40 \mathrm{MHz}$. The signals are decoded in the Digital Optical Receiver Integrated Circuit (DORIC) located on an $\mathrm{BeO}$ optoboard before being transmitted to the modules. The MCC sends events to the data acquisition system outside the detector utilizing the optoboards, where the signal is converted to an optical signal by vertical-cavity surface-emitting lasers (VCSEL) arrays. Data is then transferred via optical fibers, where the speed and modularity of the data transmission depends on the module's location on-detector. Barrel Layer- 2 modules have one data-link transmitting at 40 $\mathrm{MHz}$ while the Disks and barrel Layer-1 have one link which can run at either 40 or $80 \mathrm{MHz}$. The innermost barrel layer (B-layer), due to the high expected hit rate, has two data-links per module, which can each be read out at $80 \mathrm{MHz}$ for an equivalent readout speed of $160 \mathrm{MHz}$. The layout of the pixel readout system from the signal generation in the sensor to the off-detector RODs can be found in Fig. 3. Since beginning of 2011, the readout speed is $40 \mathrm{Mb} / \mathrm{s}$ for the Barrel Layer-2, 80 $\mathrm{Mb} / \mathrm{s}$ for the Barrel Layer-1 and disks, and $160 \mathrm{Mb} / \mathrm{s}$ for the B-Layer.

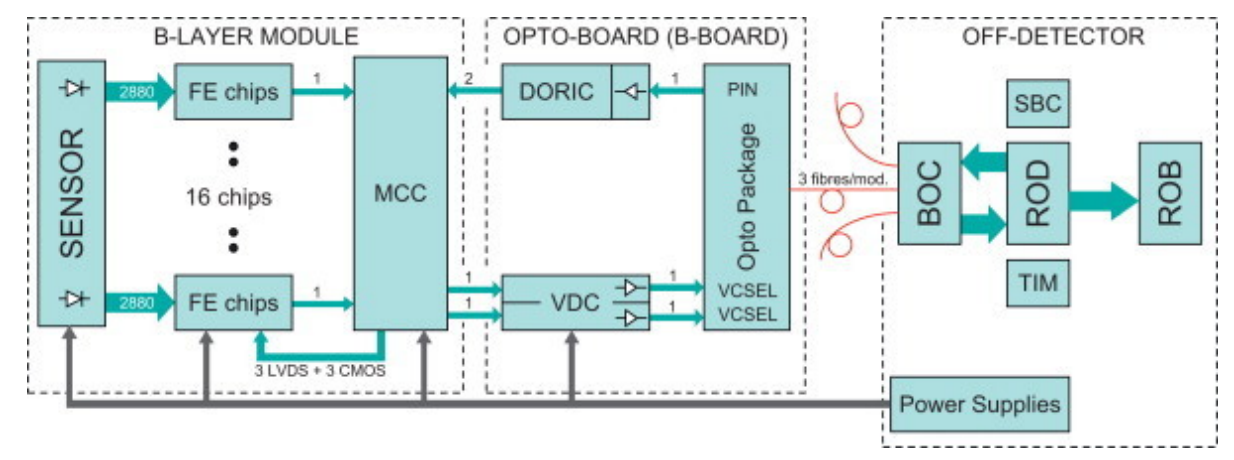

Figure 3: A schematic of the readout system for the ATLAS Pixel Detector. 


\section{Detector Operations}

\subsection{Operational Aspects}

The complete ATLAS Pixel Detector has been operated for the first time in the experiment in August 2008, shortly before the arrival of first beam in the LHC. The in-situ calibration, the noise hit masking and a crude version of the reconstruction algorithms were successfully tested and improved all along this period. Since then detector operation has continued without any major problems. Currently $96.7 \%$ of the modules are included in the ATLAS DAQ.

The non-active part contains 55 disabled modules (3.2\%) due to problems in communication or retrieval of data, open high voltage circuits, low voltage shorts, inability to receive the clock signal or failure of one optoboard. Additionally 47 single front-end chips $(0.16 \%)$ are disabled. It has been found that in particular the front-end failures are linked to thermal cycles of the detector, caused by planned or unplanned stops of the cooling plant or power cuts. A procedure for the switch on has been established, which limits temperature excursions, with first modest results. In addition to these inactive modules and front-ends, some modules may be temporarily removed from data-taking due to failures of the off-detector VCSELs which provides communication to the modules via the optical link. The failing components are accessible and can be replaced promptly.

The primary concerns for operations with beams in the LHC are to ensure the safety of the Pixel Detector, to ensure the highest data taking efficiency and to check the quality of the data during the run. During periods with unstable beams in the LHC, the Pixel Detector must be kept in a safe, standby state which requires that the sensors remain unbiased. This safety is enforced with a hardware interlock on the high voltage system which is linked to a signal sent by the LHC when beams are stable. Since the switching off of the high voltage would normally lead to a large number of noise hits per module which can block the data acquisition system (DAQ), the preamplifiers are also switched off, i.e. the sensor is decoupled from the analog portion of the pixel circuit. Under these conditions, there are no hits in the entire detector and the Pixel Detector can still be included in the ATLAS readout. A warm start procedure has been developed to enable the Pixel Detector to remain in the ATLAS DAQ system while satisfying the safety requirements. When the LHC declares stable beams, the trigger is paused and the bias voltage together with the preamplifiers are switched on. Using this procedure, the safety of the Pixel Detector is ensured and the data-taking efficiency is increased with respect to a complete restart of the ATLAS DAQ as the time necessary for the warm start procedure is of the order of several minutes while the time needed to restart the ATLAS DAQ can be significantly longer. At the end of a stable beam period the detector is brought back into a safe state automatically, reacting on either LHC handshake messages or a post mortem signal of an unplanned beam dump.

\subsection{Detector Calibration}

In order to optimize operation of the Pixel Detector, regular checks and calibrations of the working points for both the readout systems and the pixel modules have to be done. The first step is to establish stable communication through the optical links for each module. These socalled "optical scans" check that the transmitting VCSELs for each link are sending light and also identify a stable working point for the data-links in the laser-threshold/sampling-time phase 


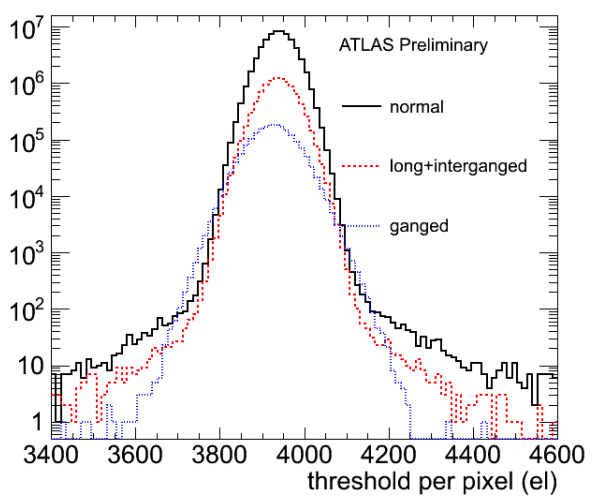

(a)

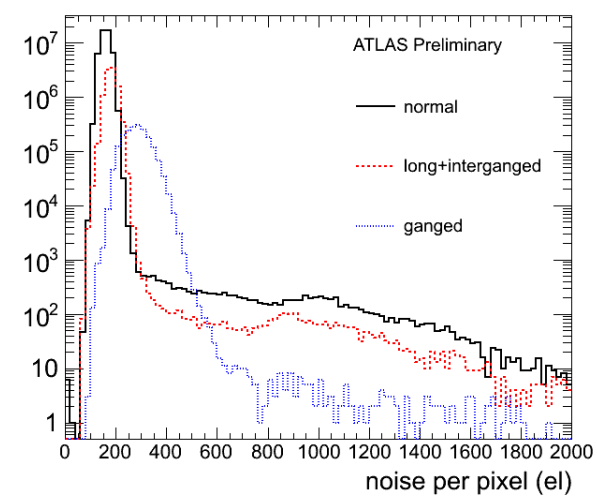

(b)

Figure 4: a) Measured threshold values for all scanned pixels in the detector. Prior to the scan the threshold settings have been tuned to $3500 e$. b) Noise values obtained from the same threshold scan.

space. Following this, the calibration parameters for the front-end electronics are measured and a parameter tuning is performed if necessary.

The target discriminator threshold for 2011 LHC collisions is 3500 electron charge equivalent (e). Each pixel is tuned to this threshold by injecting a number of test charges at target threshold into the pixel using the charge injection circuitry, varying a 7-bit DAC, determining the discriminator threshold and counting the number of generated hits. The values of threshold and noise are then extracted from an error function fit to the obtained response curve. The setting, for which the response fraction is as close as possible to $50 \%$, is the ideal setting for the given target threshold. Fig. 4a shows the measured threshold values of all scanned pixels in the detector. We see that most pixels are well-centred around the target threshold value and the typical RMS of the threshold distribution after tuning is $\sim 40 e$. The noise values obtained from the same threshold scan are shown in Fig. 4b. Typical noise values vary between $\sim 180 e$ for the normal pixels and $\sim 300 e$ for the class of the ganged pixels. Pixels exceeding a noise occupancy of $10^{-7}$ hits per bunch crossing (BC) in dedicated noise data taking runs are masked for ATLAS data taking already in the module configuration. At a threshold setting of $3500 e$ these pixels typically amount to $0.1 \%$ of all pixels.

The ToT information stored together with each hit can be used as a measure for the deposited charge. To be useful for a charge measurement the ToT has to be calibrated. This is done in a way that 30 ToT correspond to $20000 e$. This is the number of electron charge equivalents corresponding to the approximate charge deposited by a minimum ionizing particle. The resulting charge resolution is about $660 e(1 \mathrm{ToT})$. For an efficient reconstruction, the hits of particle tracks not only need to be detected, but also to be assigned to the correct bunch crossing, i.e. collision. Accounting for the trigger delays, different cable lengths and remaining module-by-module variations a dispersion of only $0.17 \mathrm{~ns}$ is achieved. Despite such a good precision, the hits from low charge depositions may end-up in the next bunch crossing, due to the slow rise of the preamplifier pulse above the discriminator threshold. This is called time-walk and leads to an uncertainty in the detection time for different signals belonging to the same collision. The critical quantity to quantify this effect 


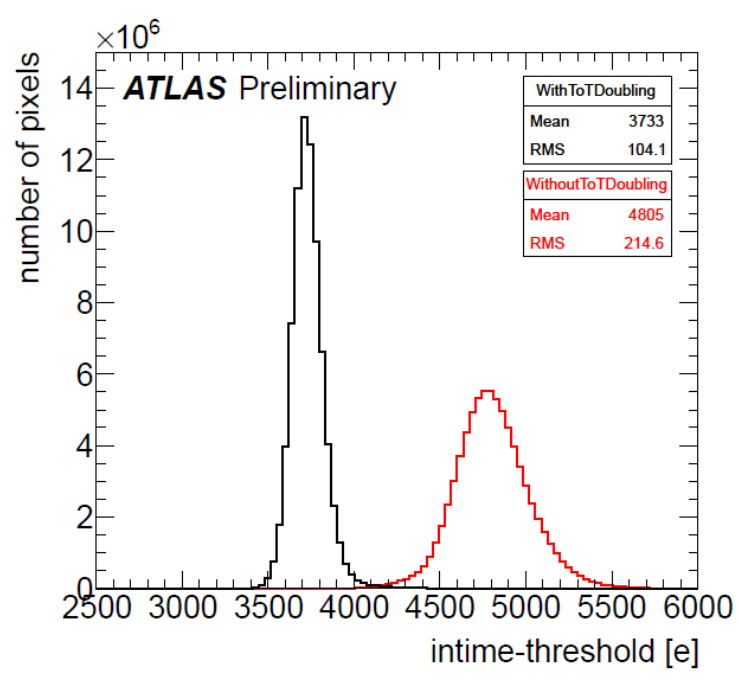

Figure 5: Measured in-time threshold for the full Pixel Detector with and without hit-doubling. All pixel were tuned to an threshold off $3500 \mathrm{e}$.

is the so-called in-time threshold, i.e. the smallest charge that is detected within the same bunch crossing as a large reference charge. A measurement of the in-time threshold values for all pixels in the detector is shown in Fig. 5. Without corrections, the average in-time threshold is 4800e, approximately $1300 e$ above the discriminator threshold of $3500 e$. Charges between $3500 e$ and $4800 e$ are detected but not assigned to the correct bunch crossing. This behaviour can be improved by a hit-doubling mechanism implemented in the FE-I3 chip. In case the ToT is below a programmable threshold, currently 6 ToT, the hit is written twice: once with the same BC time and one with the previous $\mathrm{BC}$ time. This mechanism allows to reduce the in-time threshold to a value $200-300 e$ above the discriminator threshold, as shown by the second histogram in Fig. 5, at the cost of a slight, $\sim 10 \%$, increase in the occupancy.

\section{Performance}

In order to minimize the number of hits lost to mistimed modules and hits misplaced due to the time-walk effect, the readout window of the Pixel Detector can be adjusted to record up to 16 consecutive BCs for each level-1 trigger provided by the ATLAS DAQ. At the beginning of LHC operation at a center-of-mass energy of $7 \mathrm{TeV}$ in March of 2010, the Pixel Detector used a 4 BC readout window. Through a campaign of time delay scans in the spring of 2010, the time delay per module was measured and optimized to allow for further reduction of the readout window with minimal loss in efficiency. Since October 2010, the Pixel Detector is operated with the design readout window of $1 \mathrm{BC}$.

The Pixel Detector has participated in ATLAS data-taking for all stable LHC operations with beam which began in November of 2009. Beginning in March of 2010, stable collisions have been recorded at a record center-of-mass energy of $7 \mathrm{TeV}$. While initial instantaneous luminosity was low due to a small number of filled bunches in 2009 and 2010, the LHC has made steady progress and has recently surpassed an instantaneous luminosity of $10^{33} \mathrm{~cm}^{-2} \mathrm{~s}^{-1}$. Typical occupancies at 
this luminosity are $1.5 \cdot 10^{-4}$ hits/pixel/BC for the innermost layer, $0.7 \cdot 10^{-4}$ hits/pixel/BC for Layer-1, $0.45 \cdot 10^{-4}$ hits/pixel/BC for the Layer-2, and $0.55 \cdot 10^{-4}$ hits/pixel/BC for the end-caps.

As described earlier the Pixel Detector is only taking data during stable beam runs. The Pixel Detector efficiency for data-taking has been measured to be $99.8 \%$. This efficiency is measured as the fraction of time in which the Pixel Detector is ready and active in the ATLAS DAQ for all declared stable beam periods. The luminosity-averaged efficiency for all ATLAS subdetectors can be found in Tab. 1. The dominating contribution to the Pixel Detector inefficiency is the delay for the warm start, i.e. switching on the HV and preamplifiers after the declaration of stable beams.

\begin{tabular}{ccccccc}
\hline \hline \multicolumn{2}{c}{ Inner Tracking Detectors } & Calorimeters & Muon Detectors & \multicolumn{2}{c}{ Magnets } \\
Pixel & SCT & TRT & & & Solenoid & Toroid \\
\hline $99.8 \%$ & $99.5 \%$ & $100 \%$ & $89.3 \%-99.5 \%$ & $99.5 \%-100 \%$ & $98.5 \%$ & $97.9 \%$ \\
\hline \hline
\end{tabular}

Table 1: Luminosity weighted relative detector uptime and good quality delivery during 2011 stable beams in $p p$ collisions at $\sqrt{s}=7 \mathrm{TeV}$ between March 13th and June 6th (in \%). The inefficiencies in the calorimeters will be partially be recovered in the future. The magnets were not operational for a 3-day period at the start of the data taking.

\section{Summary}

Since its commissioning in 2008, the ATLAS Pixel Detector has been successfully operated in the ATLAS experiment. Currently 97\% of the detector can be operated in physics data taking, whereas the data-taking efficiency is improved by the warm start procedure and safety is ensured by an automatic action bringing the detector back into a save state after a stable beam period. The detector behaviour is well understood in most aspects and satisfies the requirements both concerning the electronics and module behaviour and the overall tracking performance. The average pixel threshold is 3500 electrons with a dispersion of $\approx 40$ electrons. The in-time threshold value without correction is at approximately $4800 e$, using the time-walk correction of the FE chips leads to an intime threshold approximately $250 e$ above the discriminator threshold. The efficiency of the enabled modules measured in data taking with particle tracks is at $99 \%$, the spatial single-point resolution is according to the expectation. Regular calibration measurements to survey the status of the ATLAS Pixel Detector are performed.

\section{References}

[1] The ATLAS Collaboration, The ATLAS Experiment at the CERN Large Hadron Collider, JINST 3 (2008) P08003.

[2] G. Aad et al., ATLAS Pixel Detector Electronics and Sensors, JINST 3 (2008) P07007.

[3] I. Peric et al., Nucl. Instrum. Meth. A 565 (2006) 178. 